\title{
Hybrid Skeleton Driven Surface Registration for Temporally Consistent Volumetric Video
}

\author{
João Regateiro Marco Volino Adrian Hilton \\ Centre for Vision, Speech and Signal Processing \\ University of Surrey \\ \{j.regateiro, m.volino, a.hilton\} esurrey.ac.uk
}

\begin{abstract}
This paper presents a hybrid skeleton-driven surface registration (HSDSR) approach to generate temporally consistent meshes from multiple view video of human subjects. $2 D$ pose detections from multiple view video are used to estimate $3 D$ skeletal pose on a per-frame basis. The $3 D$ pose is embedded into a $3 D$ surface reconstruction allowing any frame to be reposed into the shape from any other frame in the captured sequence. Skeletal motion transfer is performed by selecting a reference frame from the surface reconstruction data and reposing it to match the pose estimation of other frames in a sequence. This allows an initial coarse alignment to be performed prior to refinement by a patch-based non-rigid mesh deformation. The proposed approach overcomes limitations of previous work by reposing a reference mesh to match the pose of a target mesh reconstruction, providing a closer starting point for further non-rigid mesh deformation. It is shown that the proposed approach is able to achieve comparable results to existing model-based and model-free approaches. Finally, it is demonstrated that this framework provides an intuitive way for artists and animators to edit volumetric video.
\end{abstract}

\section{Introduction}

Motion capture (MoCap) using optical markers is the gold standard for human performance capture, and is widely used in industry, but only captures a low-dimensional representation of the human body, i.e. joint positions/angles. It requires a huge effort from artists to create believable character animations from MoCap data. To overcome this drawback, multiple view video capture techniques have evolved towards simultaneously capturing shape, motion and appearance.

Modern capture systems generally consist of a set of synchronised cameras that simultaneously capture the scene from different viewpoints $[10,11,28]$. Feature tracking and

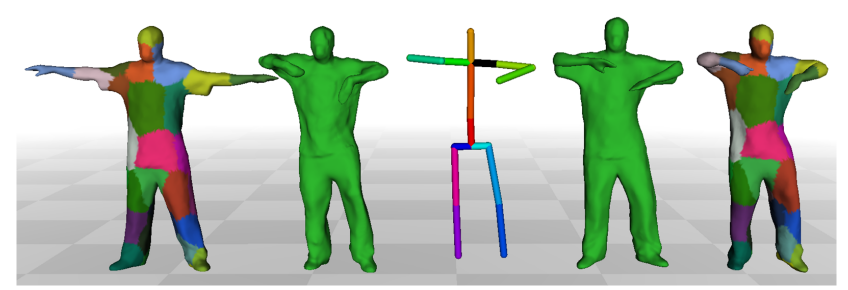

Figure 1. Processing stages of the proposed method: (left to right) Source mesh, target mesh reconstruction, 3D pose, reposing of source mesh using linear blend skinning and proposed aligned result.

stereo matching techniques are applied to the data to create a 3D reconstruction of both static and dynamic scenes. 3D Performance Capture results in a sequence of 3D geometry objects of the actor or scene, integrating all visual features, such as dynamic shape, motion, and texture appearance. However, a problem arises where for each of the captured frames the geometry is temporally inconsistent, i.e. the shape topology and vertex connectivity are varying.

Post-processing methods suffer from temporally inconsistent geometry, making it extremely difficult to propagate changes throughout a sequence. This content challenges most existing methods, as a consequence of the diversity in human motion and shape, and dynamic surfaces; it demonstrates difficulty on dense point-to-point correspondence schemes. Artists very often manually manipulate content to achieve the desired pose, or correct the geometry, spending significant work-time on such laborious task.

This work proposes an automated hybrid framework to generate temporally consistent reconstructions from dynamic mesh sequences. The core idea of the proposed approach is to embed a skeleton into a surface reconstruction and repose the shape prior to geometric alignment, see Figure 1. By performing a coarse alignment, as a pre-process, the proposed approach is able to handle large changes in pose between a reference and target shape. We also demonstrate that the resulting temporally consistent mesh representation can be used to facilitate keyframe-based editing for volumetric video. 
The contributions of this paper can be summarised as follows:

1. A 4D surface tracking framework to temporally align mesh surfaces with inconsistent topology and difference in shape. The framework successfully maps a mesh surface onto a target using only geometry information.

2. A hybrid skeleton-driven surface registration (HSDSR) to overcome the limitations of the 4D surface tracking framework, achieving more reliable and accurate results for complex mesh sequences. It provides results for longer sequences without the increase or propagation of errors generated in previous frames.

3. Keyframe-based editing for volumetric video to allow intuitive editing and propagation through a sequence, it generates novel sequences avoiding the need to recapture new datasets, maintaining surface detail and integrity.

\section{Related Work}

Human Motion Capture: Skeleton based techniques arise from the necessity of simulating human body motion. MoCap [22] estimates human movement based on tracking its skeleton using markers placed on key locations. Recent work from Wei et al. [32] introduces deep learning techniques to overcome a registration problem between complete or partial 3D models. They successfully demonstrate the benefits that deep learning offers to track human body poses with a real-time human body correspondence framework.

Convolutional Pose Machines (CPMs) [8] learn how to associate body parts to human-like shapes from a single image and estimate 2D human joints locations for multiple people. This allows more freedom on the type of data captured, because it does not rely on physical trackers, therefore the system is able to preserve all dynamics from the captured scene, such as clothing deformation and fine detail. A central challenge in computer vision over the past two decades has been markerless human motion capture from video [16, 21, 29,33].

Surface reconstruction from multiple view video capture generates unstructured mesh sequences with both the vertex connectivity and geometry changing from frame-toframe [11,28]. To overcome this issue, methods for temporal alignment of mesh sequences have been introduced to obtain sequences with temporally consistent mesh structure [4, 6, 31]. Model-based techniques allow for a better mesh representation, including fine detail and body shape characteristics, however it requires prior information computations and it cannot reproduce accurately the scene captured, such as clothes and hair dynamics [31,33]. Model-free approaches overcome limitations raised from model-based, obtaining a better representation of the captured scene, as result of not being tied to a parameterized template, however this raises problems such as, surface drifting, the accumulation of errors, and the inability of tracking two significantly different poses. Temporally inconsistent geometry does not ease the manipulation of mesh sequences, increase physical storage space, and requires a huge effort to create animation.

Geometry Alignment: Geometry alignment techniques require the establishment of correspondences between frames so it can correctly align meshes for every frame $[2,7,9,30]$. Lipman and Sorkine et al. [19, 26,34] propose a differential coordinate representation of a surface mesh, which preserves local detail information in the presence of large mesh deformations.

Huang et al. [15] presents a volumetric approach for 3D shape tracking that uses centroidal Voronoi tessellation representation to build a feature space where trained random forests return optimal correspondences. However, volumetric techniques tend to preserve the volume from frame to frame, therefore if the volume has a significant change the technique is not able to adapt. Coarse-to-fine matching demonstrates success for non-rigid registration [5, 24] across complex sequences, and is achieved by targeting subsets of a surface area and interactively decreasing its radius, preserving fine details such as dynamic clothing. These approaches focus on deforming a single reference frame through subsequent frames of the sequence, leading to the increase of artefacts on large changes in shape.

Non-sequential alignment approaches have also been investigated $[3,4,17,23]$, providing robust techniques that are able to handle larger non-rigid deformations in consecutive meshes of the reconstructed captured sequences. However, the shape comparison in the above techniques is computationally expensive and requires a full sequence as a prior, disallowing online applications.

\section{Hybrid Skeletal-driven Surface Alignment}

The objective of the proposed approach is to extract a temporally consistent mesh structure from multiple view video of a human actor. We estimate a 3D surface at each time instance $t$ using a model-free reconstruction pipeline, described in Section 3.1, resulting in a temporally inconsistent mesh representation for a captured sequence. A 2D joint detector is utilised to first estimate 2D skeletal pose in each camera view at every time instance $t$. 2D joint locations are triangulated to estimate the 3D pose using a kinematic solver, described in Section 3.2. 3D skeletal pose is used to rig the reconstruction for a reference frame from the sequence to obtain an animatable representation of the specific person and their clothing. The animated reference 


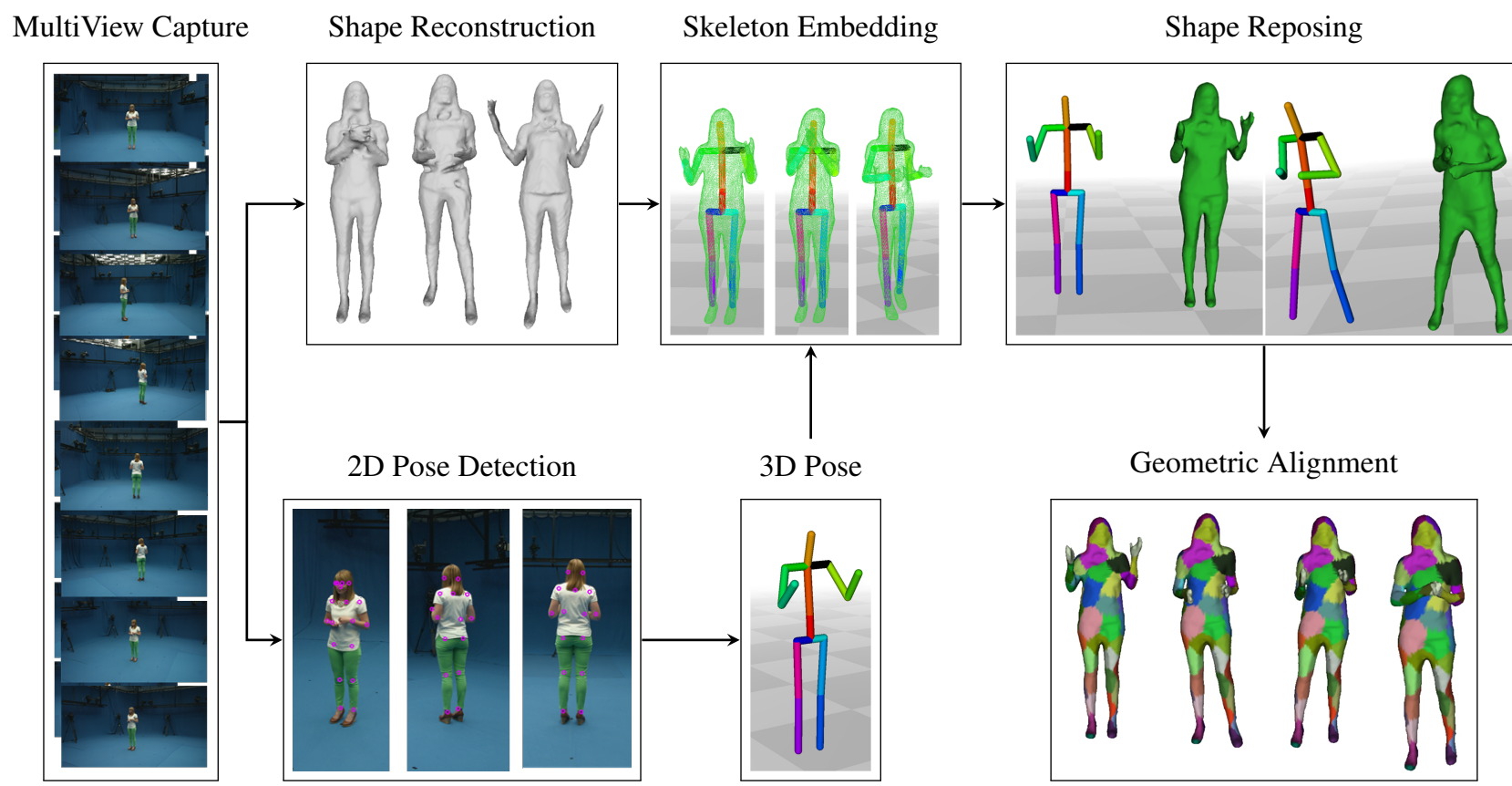

Figure 2. Proposed Hybrid Skeleton-driven surface registration pipeline, going from left to right, we have a multiple view capture system to retrieve shape and appearance. Followed by 2D pose detection and shape reconstruction, to retrieve $3 \mathrm{D}$ skeletal and surface vertex correspondence. Finalising with shape reposing to provide an initial pose estimation to the geometric alignment method.

frame is reposed using the $3 \mathrm{D}$ skeleton estimate for each frame to obtain a course alignment between the reference frame and target reconstruction, described in Section 3.3. Non-rigid patch-based Laplacian deformation is then used for detailed temporal alignment through non-rigid deformation of the reference surface, described in Section 3.4. This allows the correct alignment in sequences that include large changes in pose from frame-to-frame due to fast motion. An overview of the proposed hybrid skeleton-driven surface registration pipeline is shown in Figure 2.

\subsection{Multiple View Surface Reconstruction}

Input into our method is synchronised multiple view video $\left\{\left\{I_{c}^{t}\right\}_{c=1}^{N_{C}}\right\}_{t=1}^{N_{T}}$ recorded from $N_{C}$ calibrated cameras $\left\{C_{c}\right\}_{c=1}^{N_{C}}$, where $N_{T}$ is the number of frames, of a human actor in a controlled studio, see Figure 2. Shape reconstruction is performed by first extracting foreground silhouettes via chroma-keying allowing a visual hull [18] reconstruction at each time instance $t$. The visual hull is then refined using a volumetric graph-cut by matching stereo features across camera images $\left\{\left\{I_{c}^{t}\right\}_{c=1}^{N_{C}}\right\}$ to make the geometry photo-consistent [28]. Stereo refinement adds geometric detail to concave regions of the surface which cannot be recovered using visual hull reconstruction. This process results in a set of temporally incoherent meshes which have been independently reconstructed at each time instance.

\subsection{D/3D Pose Estimation}

2D Joint Detection: Given a set of captured images at a single time instance, $\left\{\left\{I_{c}^{t}\right\}_{c=1}^{N_{C}}\right\}$, we use a 2D pose detector to locate the skeletal joints of the subject. In the proposed pipeline, 2D joint detections are given by Convolutional Pose Machines (CPM) [8] which have been shown to be a robust $2 \mathrm{D}$ pose detector trained on a wide variety of subjects and poses. The detected 2D joint $j_{i}^{c}=\left\{p_{i}^{c}, \omega_{i}^{c}\right\}$ consists of a 2D pixel location $p_{i}^{c}$ and confidence score $\omega_{i}^{c}$.

Joint Triangulation: Triangulation of each joint requires the detected 2D joint locations of the $i^{\text {th }}$ joint from each camera $\left\{j_{i}^{c}\right\}_{c=1}^{N_{C}}$, the camera intrinsic parameters $K_{c}$, rotation matrix $R_{c}$ and translation vector $t_{c}$ for all $N_{C}$ cameras. We seek to find 3D position $\hat{j}_{i}$ for the $i^{\text {th }}$ joint that minimises the re-projection error in all camera against the detected 2D joints $\left\{j_{i}^{c}\right\}_{c=1}^{N_{C}}$. The 3D joint position $\hat{j}_{i}$ is optimised according to Equation 1. This incorporates the joint confidence $\omega_{i}^{c}$ to make it robust against errors in joint detection. In the case that a joint is not detected, $\omega_{i}^{c}=0$.

$$
\underset{\hat{j}_{i}}{\arg \min } \sum_{c=1}^{N_{c}} \omega_{i}^{c}\left\|P\left(c, \hat{j}_{i}\right)-p_{i}^{c}\right\|
$$

where $p_{i}^{c}$ and $\omega_{i}^{c}$ are the 2D detected joint location and joint confidence, respectively, for the $i^{t h}$ joint in the $c^{t h}$ camera. $P\left(c, \hat{j}_{i}\right)$ is the projection function which maps a 3D joint position into the camera image plane $P\left(c, \hat{j}_{i}\right)=K_{c} R_{c} \hat{j}_{i}+$ 
$t_{c} . \hat{j}_{i}$ is the optimised 3D position of the $i^{t h}$ joint.

The collection of 3D joints positions $\hat{j}_{i}$ represent a skeletal structure at a time instance $t$. We define a skeletal structure as containing $163 \mathrm{D}$ joint positions $\hat{j}_{i}$ and 15 bones $\hat{b}_{i j}$. The bone $\hat{b}_{i j}=\left\{\hat{j}_{i}, \hat{j}_{j}\right\}$ is the relation between the $i^{t h}$ and $j^{\text {th }}$ joints. This leads to a hierarchy relationship between bones to create a kinematic structure that allows for skeleton animation.

Kinematic Structure: A kinematic structure can be represented as a bi-directional graph, where nodes represent 3D joint positions $\hat{j}_{i}$, and edges represent bones $\hat{b}_{i j}=\left\{\hat{j}_{i}, \hat{j}_{j}\right\}$. Each node has an associated a rotation $R_{i}$ and translation $t_{i}$, defined as a transformation $T_{i}=R_{i}(\Theta) t_{i}(\nu)$, where $R_{i}(\Theta)$ is an axis-angle rotation of $\Theta$ degrees, and $t_{i}(\nu)$ is a translation of the vector $\nu$. Consequently, the joint transformation $T_{i}$ is represented as a $4 \times 4$ transformation matrix at each time instance.

Let us consider the skeletal sequence denoted $\hat{S}$ as a collection of skeletal poses that represent all the 3D joint positions $\hat{j}_{i}$ and bones $\hat{b}_{i j}=\left\{\hat{j}_{i}, \hat{j}_{j}\right\}$ for the captured sequence. Hence, a skeletal structure at a time instance $t$ is defined as $\hat{s}_{t}=\left\{\hat{j}_{i}, \hat{b}_{i j}\right\}$. The sequence $\hat{S}$ can only inform about the joints location and relationship, therefore to produce animation it is necessary to calculate the joint transformations that maps one frame $\hat{s}_{t}$ into $\hat{s}_{t+1}$. The kinematic structure transforms an initial pose $\theta$ onto $\hat{s}_{t}$, translating the motion from the captured sequence $\hat{S}$ to kinematic skeletal joint motion. The initial pose $\theta$ is a kinematic structure, composed of 16 nodes $N_{N}$ and 15 edges $N_{B}$. Each edge has nodes associated, describing the dependency between nodes; nodes contain a transformation matrix $T_{i}$ to calculate their spatial transformation from frame-to-frame relative to its parent.

Kinematic Motion: To represent kinematic motion, we select an initial frame $\hat{s}_{t}$ and initialise pose $\theta$ as the reference pose. This work selects the first frame of the sequence as the initial pose $\theta$. Once $\theta$ is solved for the first $\hat{s}_{t}$ frame, we calculate an initial list of nodes transformation $T_{i}$ that relate to the first frame. Consequently, it is possible to map $\theta$ into any $\hat{S}$ given the initial estimation of the position and orientation. The pose $\theta$ is solved by calculation node transformations that satisfy the constraints given by $\hat{s}_{t}$.

The node transformation is solved using an inverse and forward kinematic chain approach, Equation 2, where $\rho\left(\hat{b}_{B}\right)$ is a set of parent nodes of the bone $\left\{\hat{b}_{b}\right\}_{b=1}^{N_{B}}=\hat{b}_{i j}$, and $\left[R_{\hat{b}_{b}} \mid t_{\hat{b}_{B}}\right]$ is the transformation matrix of the bone $\hat{b}_{B}$, forward kinematics uses the opposite direction of transformation, i.e. the transformation is propagated from a node to all its descendents. Firstly, the global transformations of key joints are retrieved, using the hip joint as the root transformation relative to the initial pose $\theta$. Secondly, we identify the rotation of the upper body, i.e. the rotation of the shoulders given by its parent joint - the neck in this instance. Finally, we retrieve the head orientation to be able to change the head rotation independent from the rest of the body. The remaining limbs are solved using inverse kinematics to give an initial estimate of their possible locations using the extremity joint as end-effectors. This approach allows for errors caused by incorrect or non-existent 2D detection.

The final step is a refinement of joint positions using forward kinematics to eliminate ambiguities caused by the previous step. The different skeleton poses are represented with the initial pose $\theta$ and a collection of node transformations $T_{i}$ which allows the reposing of $\theta$ to all frames in $\hat{S}$. For every frame $\hat{s}_{t} \in \hat{S}$, pose $\theta$ is mapped onto $\hat{s}_{t}$ using $\theta=\varrho\left(\hat{s}_{t}, T_{i}\right)$, where $\varrho\left(\hat{s}_{t}, T_{i}\right)$ solves $T_{i}$ for the new set of $\hat{s}_{t}$ constraints using an inverse and forward kinematic chain approach.

$$
\tau\left(\hat{b}_{b}\right)=\Pi_{\hat{b}_{b} \in \rho\left(\hat{b}_{b}\right)}^{N_{B}}\left[\begin{array}{c|c}
\mathrm{R}_{\hat{b}_{b}} & \mathrm{t}_{\hat{b}_{b}} \\
0 & 1
\end{array}\right]
$$

This gives a sequence of skeletal poses based on joint rotations from the estimated skeletal joint positions obtained by combining multiple view 2D pose using equation 1

\subsection{Skeleton Embedding and Shape Reposing}

Rigging and animating a reference frame mesh is achieved using an automated rigging framework [1]. Given a template reference frame and its skeleton reference pose, skinning weights for every vertex are estimated according to the set of nearest bones. Once the weights are calculated, we choose to use linear blend skinning (LBS) to repose the reference mesh. The animation framework receives as input an initial pose $\theta$, a collection of joint transformations $T_{i}$ and the vertex weights. This allows the reference mesh to be reposed according to the detected $3 \mathrm{D}$ pose for each frame in the sequence to obtain an initial coarse mesh approximation of the surface shape at that frame. This is then refined through geometric alignment to represent the detailed shape for each frame.

\subsection{Geometric Alignment}

This section describes a pipeline that converts an unstructured mesh sequence into a temporally consistent mesh sequence. The pipeline takes as input the reposed reference mesh obtained from the animation framework, Section 3.3. Temporally consistent meshes are produced by optimising for rigid-registration and non-rigid deformation using a Laplacian deformation.

Rigid Surface Registration: Geometric surface registration receives as input the initial animation mesh vertices $N_{P}$, and will iteratively find an optimal rigid transforma- 
tion $T_{p}=\left\{R_{p}, t_{p}\right\}_{p=1}^{N_{P}}$, where $R_{p}$ is a $3 \times 3$ rotation matrix and $t_{p}$ is a translation that aligns two sets of $3 \mathrm{D}$ vertices $\left\{P_{p}\right\}_{p=1}^{N_{P}}$ defined as patches. The following section describes the approach taken to refine transformation that overlaps the two data sets: Patch-based Iterative Closest Point $(\mathrm{PbICP})$ is employed to estimate the rigid transformation from nearest point correspondence $[4,6]$.

\section{Patch-based Iterative Closest Point Framework: PbICP} consists of three processes: ICP, registration and correspondence search. The objective of this framework is to guarantee the best fitting between a patch and a target mesh. Patches are generated using a geodesic variant of the Lloyd's algorithm [20] which partitions the mesh into hierarchy of regions, and reshapes the data until it has uniformly-sized convex cells.

The patches maintain local mesh detail and allow for re-positioning onto the target surface, thus creating a new pose with minimal loss in surface detail. The registration is performed using a variant of ICP $[14,25,35]$ represented by Equation 3, and guarantees to return the best transformation between two meshes. The correspondence search computes the closest point from all source $N_{A}$ to target $N_{F}$ vertices within the patches, and vice-versa, taking into account the vertex normals and distances. Thus, the search area is constrained to be the best approximation to the patch. To stop the rigid transformation from drifting, a constraint on the correspondence search is introduced, rejecting vertex matches where the difference in normal angle is greater than $50^{\circ}$.

$$
E=\arg \min _{R_{p}, t_{p}} \sum_{a=1}^{N_{A}} \min _{f \in N_{F}} w_{a}\left\|R_{p} p_{p a}+t_{p}-q_{f}\right\|^{2}
$$

where $w_{a}$ is the weight corresponding to the residual distance between source $\left\{p_{p a}\right\}_{a=1}^{N_{A}}$ and target points $\left\{q_{f}\right\}_{f=1}^{N_{F}}$.

Equation 3 estimates an optimal transformation that minimises the error for a patch has been found. This is done by re-calculating the closest points on every iteration, allowing the patch to find different correspondences throughout the fitting process.

Laplacian Deformation Framework: The Laplacian consists of differential coordinates to represent a mesh surface [19,34]. Differential coordinates [12,13] represent local geometric detail. The PbICP results in an approximation of the target mesh, although rigid displacement tends to cause artefacts and remove the integrity. The Laplacian deformation regularises the mesh to its original shape condition and fit to the target mesh. To solve the Laplacian deformation problem, we first constrain a group of vertices so we can find a least squares solution for the unconstrained vertices, allowing the mesh $M$ to deform.

$$
\overrightarrow{v_{u}}=\arg \min _{v_{u}}\left\|L v_{u}-\delta\left(v_{k}\right)\right\|^{2}+\left\|W_{c}\left(v_{u}-v_{k}\right)\right\|^{2}
$$

where $v_{u}$ is the set of unknown deformed vertex locations to be computed and $v_{k}$ is the known vertex locations. The vertices $v_{k}$ can also represent hard constraints, depending on if the vertex is predefined to be exactly transformed to its target position. Consequently each element of $v_{k}$ is defined with the following constraints.

$$
v_{k}=\left\{\begin{array}{c}
v_{k} \text { if the location of the } k^{t h} \text { vertex is known } \\
0 \text { otherwise }
\end{array}\right.
$$

The rigidity of the mesh is defined as $\left\|L v_{u}-\delta\right\|^{2}$, allowing the mesh surface to be manipulated onto the target shape. The preservation of surface detail is described by a constraint energy as $\left\|W_{c}\left(v_{u}-v_{k}\right)\right\|^{2}$, providing uniform weighting along the mesh surface, preserving detail and shape. The variable $W_{c}$ represents the diagonal weight matrix, where each position on its diagonal is 1 if the corresponding vertex is constrained, and 0 otherwise. To solve the location for all vertices based on soft constraints, Equation 4 is minimised with respect to $v_{u}$. The Laplacian operator $L=G^{T} D G$, where $G$ is the discrete gradient operator, and $D$ is the diagonal matrix of triangle areas and $\delta\left(v_{k}\right)$ is the differential coordinates of $v_{k}$ [27].

\section{Results and Evaluation}

This section presents results and evaluation for the proposed hybrid skeletal-driven surface registration, presented in section 3 compared with previous model-based and model-free alignment approaches.

Error Metric: Evaluation is performed using one-sided Hausdorff distance defined as $H_{B}(A)=\sup _{a \in A} d(a, B)$, where $d(a, B)$ is the distance from a point $a$ to a set $B$, which has shown good measurements between two 3D meshes. The comparison was performed between the final temporally consistent result and the target mesh reconstruction at each time instance. We have evaluated our results against both model-based and model-free approaches.

Model-based: We have measured the results against two publicly available sequences from Vlasic et al. [31], Samba and Crane datasets (Figure 3 row 1 and row 2) to the target mesh reconstruction and compared with the proposed results, see Figure 4 and Figure 5. From Figure 7, it is visible that the mean error is smaller then $1 \mathrm{~cm}$, demonstrating the ability to maintain local fine details on loose clothing sequences. Comparing with Vlasic datasets we were able to maintain the same human pose and maintain the clothing dynamics tracked from the proposed pipeline. 

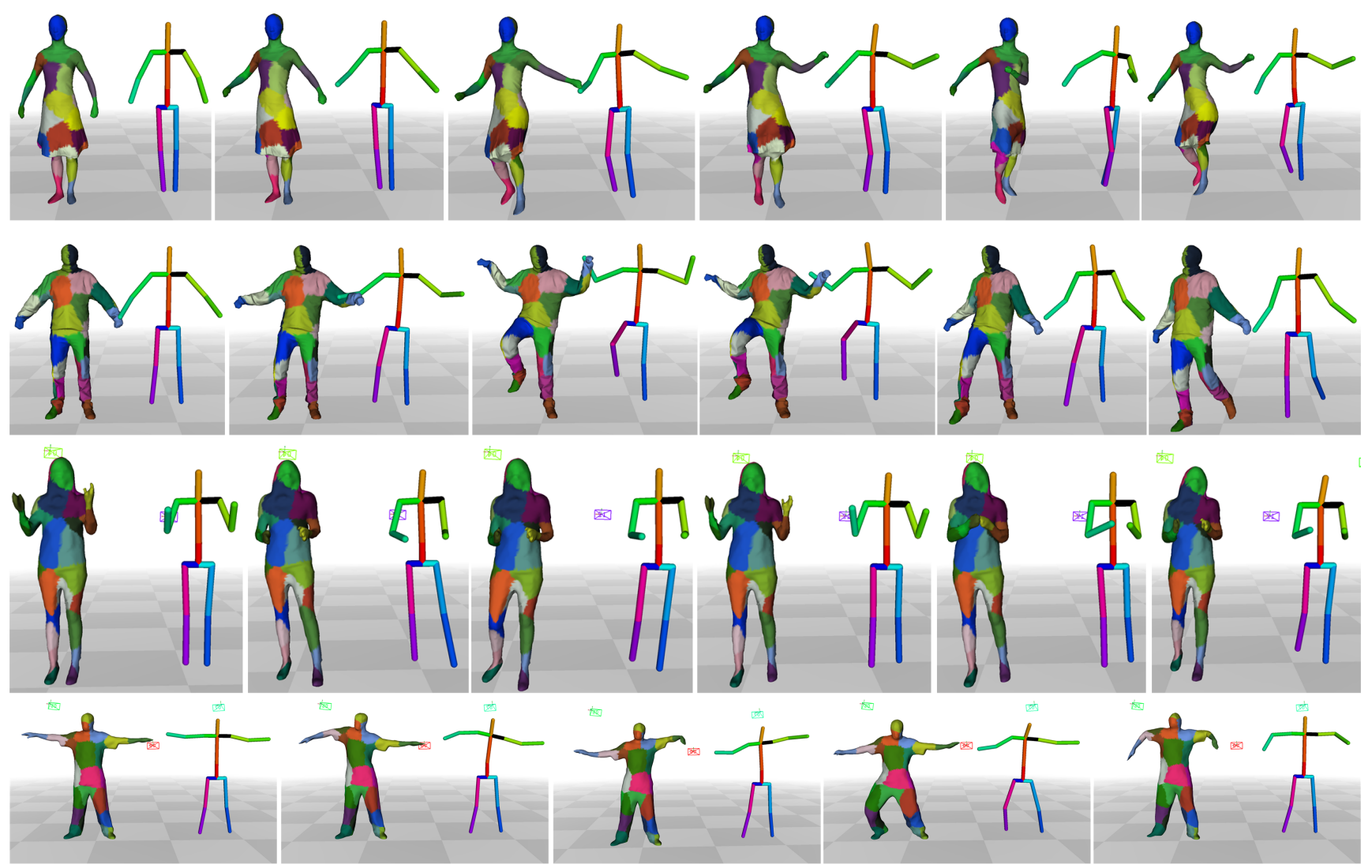

Figure 3. Proposed results on four sequences, from top to bottom, Vlasic samba and crane [31], Rachel dataset, and SurfCap street dance dataset [28]. From left to right the images illustrates temporally consistent meshes across a sequence and its respective tracked pose at that time instance.

Model-free: The Rachel sequence was captured to demonstrate difficulties with body parts being in close contact (Figure 3 row 3). We have measured the proposed result to the target mesh reconstruction to demonstrate the minimal residual error for sequences where the subject is in skin tight clothing with body contact. This demonstrates the ability to have mesh surface contact without loosing tracking or causing undesired artefacts. From Figure 7, it is visible that the mean error is smaller then $1 \mathrm{~cm}$, demonstrating the ability to maintain local fine details on loose clothing sequences.

We have also compared the proposed methos to results from the method proposed by Budd et al. [4] on the SurfCap JP street dance dataset [28], to the target mesh reconstruction (see Figure 3 row three). Comparing with the method of Budd et al. [3], we were able to maintain the same human pose and maintain the clothing dynamics tracked from our reconstruction methods.

Performance: Presented results were generated using a desktop PC with an Intel i7-2600 CPU 3.40GHz, 12GB of RAM and a Nvidia Geforce GTX 960 GPU. Run-time per- formance varies based on the number of cameras and mesh complexity. Per-frame computation times for the evaluation datasets are as follows: Vlasic [31] (8 cameras, 10k vertices) 30 seconds, SurfCap [28] (8 cameras, 17k vertices) 3 minutes, and Rachel (16 cameras, $11 \mathrm{k}$ vertices) 30 seconds.

Limitations: The limitations of the proposed approach are in two areas: joint detection and 3D pose errors caused by axial rotation. Failure of the pose detection typically occurs where observed poses are not represented in the training data, e.g. handstands and complex self-occlusion. Given an incorrect 3D pose estimation, the reposed reference mesh may not be sufficiently close to the target surface to find correspondence. The second limitation comes from the inability to accurately solve for axial rotation, e.g. wrist rotation. This can result in 'candy wrapper' artefacts occurring when the reference mesh is reposed.

\section{Applications}

Mesh Compression: Temporal alignment of dynamic mesh sequences enforce a consistent mesh topology over a sequence. This reduces storage and transmission require- 


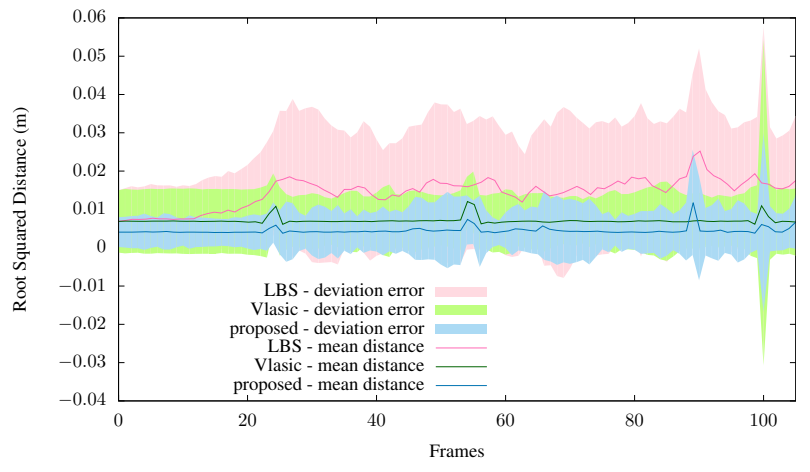

Figure 4. The graph shows the mean distance and the standard deviation from result to target mesh of Vlasic Samba performance sequence.

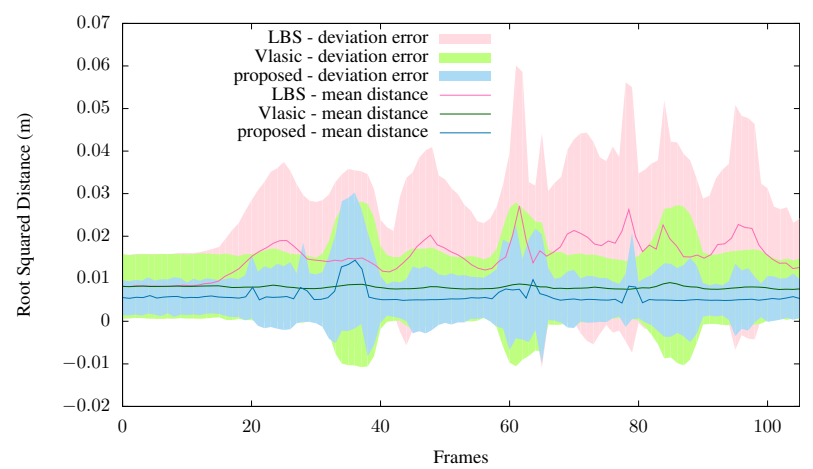

Figure 5. The graph shows the mean distance and the standard deviation from result to target mesh of Vlasic Crane performance sequence.

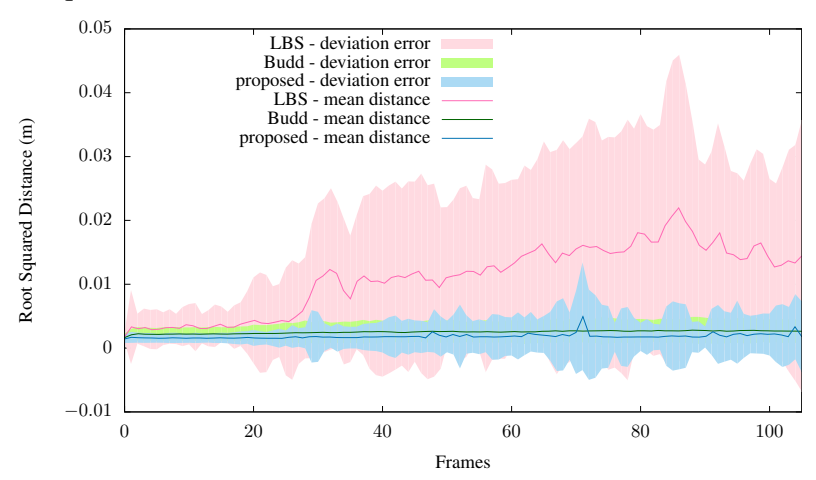

Figure 6. The graph shows the mean distance and the standard deviation from result to target mesh of JP pop performance sequence.

ments as only vertex positions change on a per frame basis and attributes such as mesh connectivity and texture coordinates remain constant.

Editing of Volumetric Video: The proposed approach enables artistic editing of volumetric video through a keyframe-based animation framework. This allows manipulation of volumetric video in a way that is intuitive to artists and compatible with existing animation frameworks. This also provides the opportunity for correction and extension of the captured motions without the need for additional data captures saving on cost.

Figure 10 illustrates example edits on two sequences. First, we demonstrate adjustments in head orientation and arm pose on the Vlasic Samba dataset [31]. The second set of images demonstrate keyframe-based animation on the Rachel dataset. In this edit the knee of the character was raised at a keyframe. This edit was propagated to surrounding frames to give a smooth and natural motion.

\section{Conclusions and Future Work}

The proposed hybrid skeleton-driven surface registration approach allows a temporally consistent mesh representation to be computed from multiple view video of human subjects. We improve on existing methods by first embedding a 3D skeleton into the estimated surface geometry. Using LBS a reference frame selected from the sequence can be reposed to match the pose of any other frame in the sequence prior to non-rigid geometry alignment. This provides a closer starting point for performing patch-based non-rigid geometry alignment. In particular, this reduces errors in geometry correspondence caused when the reference and target surface vary substantially.

We have shown the proposed method is able to track motion from various public datasets that include loose clothing and fast motion. We have also shown that this framework can be used to edit volumetric video by simply setting keyframes based on solved 3D pose. This is an intuitive method employed by animators to edit traditional CGI elements and would allow volumetric video to fit into existing pipelines.

The framework was implemented with a modular design to allow future improvements in targeted areas. For instance, geometry correspondence could be improved by introducing more information from the captured scene, e.g. colour information in situations where the colour pattern varies throughout the surface. 2D joint detection and skeleton tracking can be improved by using more training data in situations where subjects perform unusual movements that cause self occlusion such as holding other parts of the body.

Future work will focus on extending this approach to correctly identify multiple subjects and track them through time, opening the possibility for more complex multipleperson scenes.

\section{Acknowledgements}

This research was supported by the EPSRC AudioVisual Media Research Platform Grant (EP/P022529/1) and InnovateUK project Total Capture (102685). The authors would also like to thank Charles Malleson for useful discussions. 


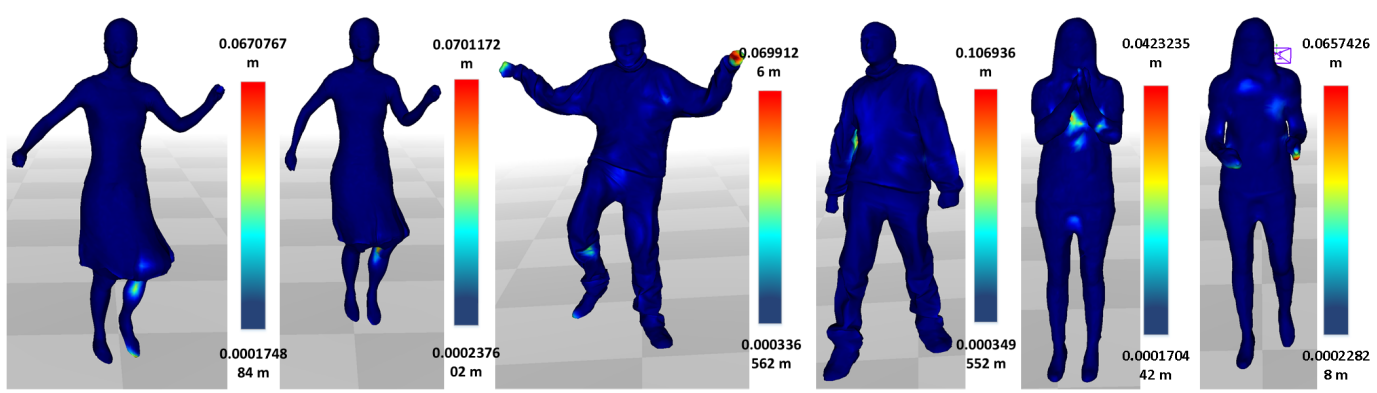

Figure 7. Illustrate error with respect to stereo reconstruction. The error is the vertex distance from result to stereo reconstruction measured in meters. The colour scheme show the variation from the minimum (blue) to the maximum (red) error.

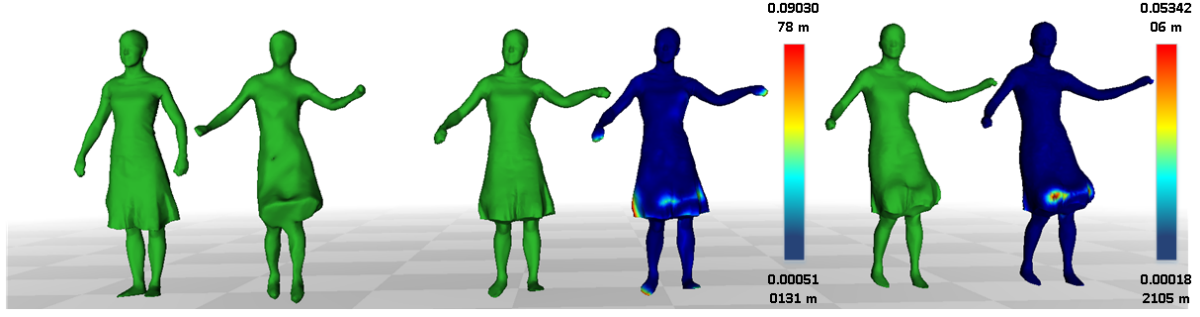

Figure 8. Comparison of the results of performing Linear Blend Skinning against using the proposed pipeline. From left to right, the source mesh, target mesh, Linear Blend Skinning to the target frame, heat map showing error with respect to the stereo reconstruction, proposed result and heat map showing error with respect to the stereo reconstruction.

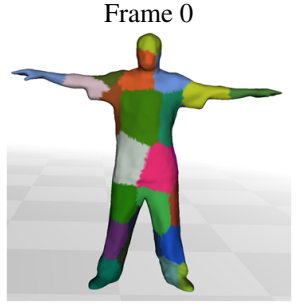

Reference

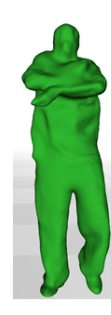

Target
Frame 123

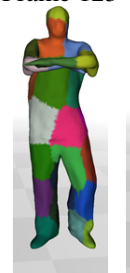

Result with reposing

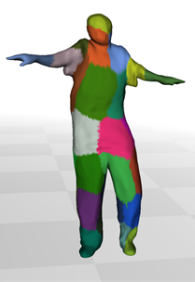

reposing

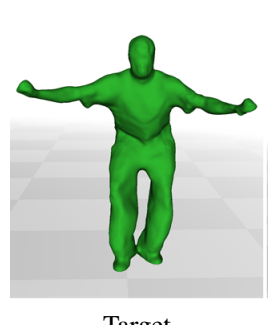

Target
Frame 160

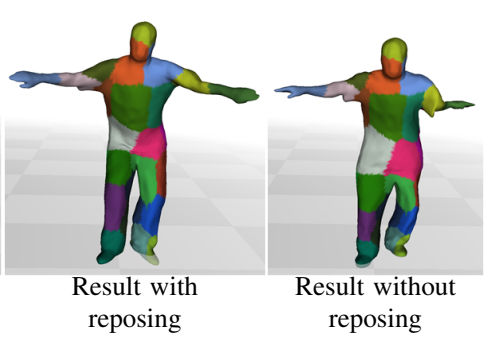

Figure 9. Comparison against surface alignment technique using the SurfCap dataset [28]. The first image from the left represents the initial template. There are two set of results, frame 123 and frame 160 showing significant differences in pose. On both frames from left to right, we have the target reconstruction, followed by the proposed result and finishing with the result without skeletal assistance.
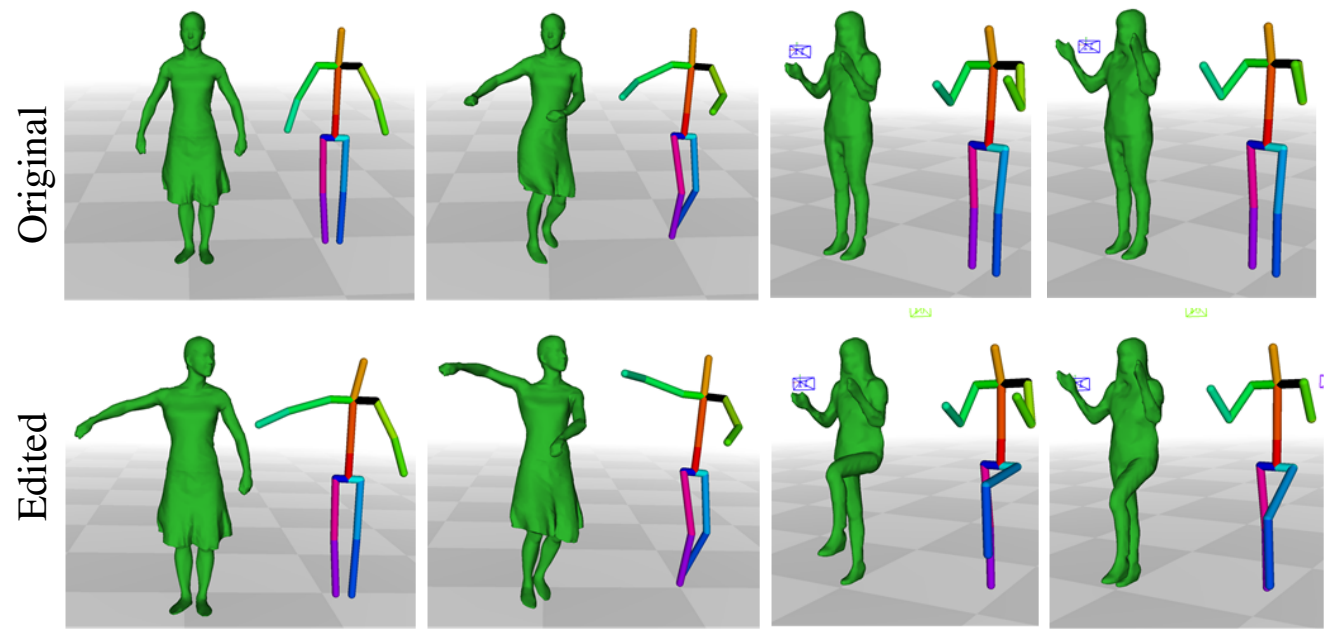

Figure 10. Illustration of frame editing, the top row of images are the original frames and the bottom row are the edited frames. 


\section{References}

[1] I. Baran, J. Popović, I. Baran, and J. Popović. Automatic rigging and animation of $3 \mathrm{D}$ characters. In ACM SIGGRAPH 2007 papers on - SIGGRAPH '07. ACM Press, 2007.

[2] A. M. Bronstein, M. M. Bronstein, and R. Kimmel. Calculus of Nonrigid Surfaces for Geometry and Texture Manipulation. IEEE TVCG, 2007.

[3] C. Budd, P. Huang, and A. Hilton. Hierarchical Shape Matching for Temporally Consistent 3D Video. In 2011 International Conference on 3D Imaging, Modeling, Processing, Visualization and Transmission. IEEE, 2011.

[4] C. Budd, P. Huang, M. Klaudiny, and A. Hilton. Global Nonrigid Alignment of Surface Sequences. IJCV, 2013.

[5] C. Cagniart, E. Boyer, and S. Ilic. Iterative mesh deformation for dense surface tracking. In 2009 IEEE 12th International Conference on Computer Vision Workshops, ICCV Workshops. IEEE, 2009.

[6] C. Cagniart, E. Boyer, and S. Ilic. Free-form mesh tracking: A patch-based approach. In 2010 IEEE Computer Society Conference on Computer Vision and Pattern Recognition. IEEE, 2010.

[7] C. Cagniart, E. Boyer, and S. Ilic. Probabilistic deformable surface tracking from multiple videos. In Proceedings of the 11th European Conference on Computer Vision: Part IV, ECCV'10, Berlin, Heidelberg, 2010. Springer-Verlag.

[8] Z. Cao, T. Simon, S.-E. Wei, and Y. Sheikh. Realtime Multi-person 2D Pose Estimation Using Part Affinity Fields. In 2017 IEEE Conference on Computer Vision and Pattern Recognition (CVPR). IEEE, 2017.

[9] D. Casas, M. Tejera, J.-Y. Guillemaut, and A. Hilton. Interactive Animation of 4D Performance Capture. IEEE Transactions on Visualization and Computer Graphics, 2013.

[10] A. Collet, M. Chuang, P. Sweeney, D. Gillett, D. Evseev, D. Calabrese, H. Hoppe, A. Kirk, and S. Sullivan. Highquality streamable free-viewpoint video. ACM Trans. Graph., 2015.

[11] E. de Aguiar, C. Stoll, C. Theobalt, N. Ahmed, H.-P. Seidel, and S. Thrun. Performance capture from sparse multi-view video. ACM Transactions on Graphics, 27, 2008.

[12] M. P. Do Carmo. Differential Geometry of Curves and Surfaces : Revised and Updated Second Edition. Dover Publications, 2016.

[13] M. Fiedler. Czechoslovak Mathematical Journal Miroslav Fiedler Algebraic connectivity of graphsa algebraic connectivity of graphs). Czechoslovak Mathematical Journal, 1973.

[14] B. K. P. Horn. Closed-form solution of absolute orientation using unit quaternions. Journal of the Optical Society of America A, 4, 1987.

[15] C.-H. Huang, B. Allain, J.-S. Franco, N. Navab, S. Ilic, and E. Boyer. Volumetric 3D Tracking by Detection. In 2016 IEEE Conference on Computer Vision and Pattern Recognition (CVPR). IEEE, 2016.

[16] C.-H. Huang, E. Boyer, and S. Ilic. Robust Human Body Shape and Pose Tracking. In 2013 International Conference on $3 D$ Vision. IEEE, 2013.
[17] P. Huang, C. Budd, and A. Hilton. Global temporal registration of multiple non-rigid surface sequences. In CVPR 2011. IEEE, 2011.

[18] A. Laurentini. The visual hull concept for silhouette-based image understanding. IEEE Transactions on Pattern Analysis and Machine Intelligence, 1994.

[19] Y. Lipman, O. Sorkine, M. Alexa, D. Cohen-Or, D. Levin, C. Rössl, and H.-P. Seidel. Laplacian Framework for Interactive Mesh Editing. International Journal of Shape Modeling, 2005.

[20] S. Lloyd. Least squares quantization in PCM. IEEE Transactions on Information Theory, 1982.

[21] C. Malleson, M. Volino, A. Gilbert, M. Trumble, J. Collomosse, and A. Hilton. Real-time full-body motion capture from video and imus. In $3 D V, 2017$.

[22] A. Menache. Understanding Motion Capture for Computer Animation, Second Edition. Morgan Kaufmann Publishers Inc., San Francisco, CA, USA, 2nd edition, 2010.

[23] A. Mustafa, H. Kim, and A. Hilton. 4d match trees for nonrigid surface alignment. In $E C C V, 2016$.

[24] M. Rouhani, E. Boyer, and A. D. Sappa. Non-rigid Registration Meets Surface Reconstruction. In 2014 2nd International Conference on 3D Vision. IEEE, 2014.

[25] S. Rusinkiewicz and M. Levoy. Efficient variants of the ICP algorithm. In Proceedings Third International Conference on 3-D Digital Imaging and Modeling. IEEE Comput. Soc.

[26] O. Sorkine. Differential Representations for Mesh Processing. Computer Graphics Forum, 2006.

[27] O. Sorkine and M. Alexa. As-rigid-as-possible Surface Modeling. Eurographics Association, 2007.

[28] J. Starck and A. Hilton. Surface capture for performancebased animation. IEEE Computer Graphics and Applications, 2007.

[29] C. Stoll, N. Hasler, J. Gall, H.-P. Seidel, and C. Theobalt. Fast articulated motion tracking using a sums of Gaussians body model. In 2011 International Conference on Computer Vision. IEEE, 2011.

[30] T. Tung and T. Matsuyama. Dynamic surface matching by geodesic mapping for 3D animation transfer. In 2010 IEEE Computer Society Conference on Computer Vision and Pattern Recognition. IEEE, 2010.

[31] D. Vlasic, I. Baran, W. Matusik, and J. Popović. Articulated mesh animation from multi-view silhouettes. In ACM SIGGRAPH 2008 Papers, SIGGRAPH '08. ACM, 2008.

[32] L. Wei, Q. Huang, D. Ceylan, E. Vouga, and H. Li. Dense Human Body Correspondences Using Convolutional Networks. In 2016 IEEE Conference on Computer Vision and Pattern Recognition (CVPR). IEEE, 2016.

[33] W. Xu, A. Chatterjee, M. Zollhöfer, H. Rhodin, D. Mehta, H. Seidel, and C. Theobalt. Monoperfcap: Human performance capture from monocular video. ToG, 2018.

[34] L. Yaron, S. Olga, C.-O. Daniel, L. David, R. Christian, and S. Hans-Peter. Differential Coordinates for Interactive Mesh Editing. IEEE Computer Society, 2004.

[35] Z. Zhang. Iterative Closest Point (ICP). In Computer Vision, pages 433-434. Springer US, Boston, MA, 2014. 